\title{
Consistent services throughout the week for acute medical care
}

\author{
${ }^{1} \mathrm{~S}$ Dunn, ${ }^{2} \mathrm{M}$ Jones, ${ }^{3} \mathrm{~T}$ Woodcock, ${ }^{4} \mathrm{~F}$ Cullen, ${ }^{5} \mathrm{D}$ Bell, ${ }^{6} \mathrm{~J}$ Reed \\ ${ }^{1}$ Programme Manager, RCPE; ${ }^{2}$ Director of Standards, RCPE and Clinical Director Durham University Hospitals Trust; ${ }^{3}$ Public Health and \\ Information Intelligence Co-Lead, NIHR CLAHRC NWL; ${ }^{4}$ Project Officer, NIHR CLAHRC NWL; ${ }^{5}$ President, RCPE; ${ }^{6}$ Deputy Director and \\ Academic Theme Lead, NIHR CLAHRC NWL
}

\section{INTRODUCTION}

At Durham University on 22 September 2015, the Royal College of Physicians of Edinburgh, in collaboration with the Centre for Healthcare Improvement and Research, hosted an expert workshop on Consistent Services for Acute Medical Care. The invited expert audience included representatives from a broad range of healthcare professions, commissioners, researchers, policy and patient communities.

The objective of the session was to take a whole systems look at the provision of consistent care throughout the working week, including out of hours weekends, with a focus on acute care. This provided clear parameters for the discussion while covering the majority of patients admitted to hospital as an emergency. This group of patients has a recognised morbidity and mortality and are known to require multi-professional care. The workshop had three linked component phases: to develop and design an action effect diagram (AED)' to clarify aims; to review evidence; and to capture a picture of patient and staff experience.

\section{SESSION I - ACTION EFFECT DIAGRAM}

The group developed an AED (Appendix A, via link at the end) with a clear aim as the starting phase of the project to capture and theme the elements of care considered necessary for a high quality acute medical care system. The AED produced used the NHS London Quality Standards for acute emergency services as a backbone for the expert workshop, being the most comprehensive published standards available. This allowed the components required to deliver consistent high quality acute care to be mapped to produce an agreed vision for the future delivery of care. This methodology ensured that the patient was central and that outputs were inclusive of the range of professional groups and departments involved in acute care. Further work has been done to develop the AED through a service and staff exemplar output. More recently, NICE Reviews and further evidence have been published and are discussed below. The group recognised that change was necessary while acknowledging the need to ensure that appropriate care is given within sustainable systems and services. This included assessing the potential impact on financial and human resources. There was an emphasis on the need for an evidence based approach to help determine which elements should be prioritised.

The group highlighted key recommendations including:

- Solutions to consistent acute medical care will be multi-professional and based on the building blocks of robust research, data and analysis

- Key services include medical, nursing, allied health, pharmacy and diagnostics with appropriate access to specialty, mental health and community and social care

- A phased move towards consistent services will maintain a sustainable quality of care

\section{SESSION 2 - WHAT DO THE DATA TELL US?}

Reviews of published papers informed the discussion. Two presentations covered the current data at an international level with an exploration of hospital unit derived data. Professor Paul Aylin focused on outputs from big data sets and Mr Neil Pettinger examined the weekday effect on patient flow through acute medical units highlighting differences in lengths of stay and bed occupancy indicators.

Professor Aylin undertook an overview of the evidence on weekend outcome data in relation to mid-week care with an examination of the strengths and limitations of a number of published studies. Limitations of the data were discussed with a clear message that small or single centred studies are underpowered and hence less likely to show poorer outcomes at weekends. Factors affecting outcomes and the conflicting evidence ${ }^{2}$ were discussed. Those which may influence weekend outcomes are likely to be complex. These include potential variation in case mix and illness severity, healthcare professional grades and staffing ratios (particularly including nursing), timeliness of access to diagnostics and seniority of attending physicians. In combination, these form an interactive set of factors that will potentially influence increased 30-day mortality rates for out-of-hours admissions. In contrast to some previous publications, the recently published Freemantle analysis ${ }^{3}$ suggests that the case mix and illness severity $y^{4}$ is indeed a higher risk at weekends when there is reduced access to services. However, there is also evidence that suggests the 30-day 
Average length of AMU stay by day of week admitted

(Large hospital calendar year 20I4 acute medical unit)

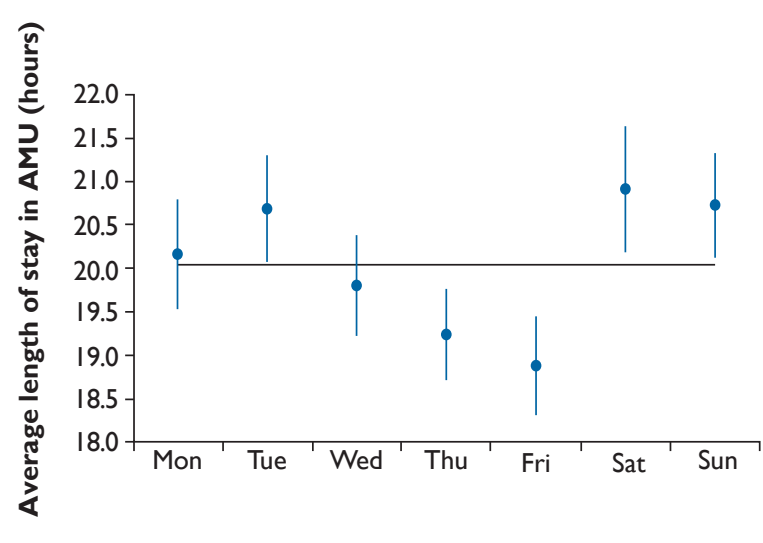

CHART I Average length of stay by day of week admitted in large acute medical unit

mortality rate for hospital admissions Friday to Monday is increased by up to $15 \%$ prior to adjustment for case mix. However, Professor Aylin highlighted that this is based on a $1.8 \%$ risk of death overall and, in context, does remain a relatively low risk. ${ }^{5}$

A European study by Aiken et al. on nurse staffing and education and hospital mortality ${ }^{6}$ found a $7 \%$ increase in the likelihood of 30-day mortality for every increase of one patient in a nurses' workload; and for every $10 \%$ increase in bachelor's degree nurses, there was a $7 \%$ decrease in mortality as an outcome. A study by Bray et al. also found an association between registered nursing staff ratios and stroke patient mortality. In January 2016, after this workshop took place, NICE released four evidence reviews on safe staffing. ${ }^{8}$ A review into Safe Staffing for Nursing in a related specialty found weak evidence, with some association between nurse staffing levels, care time and time to leaving the department. ${ }^{9}$ It is now for NHS Improving Quality to continue this work.

There remains conflicting evidence on the scale and causes of the weekend effect; however, it is a collective responsibility to consider this in all its complexity in order to arrive at sound and evidence-based action; and to recognise the roles of all professional groups contributing to acute medical care.

\section{SESSION 3 - PATIENT AND STAFF EXPERIENCE}

Delegates undertook an emotional mapping exercise to assess perceptions of experience of care at different times during the week as well as to ensure a patient focus for the ongoing discussions.

Delegates described in one word what they believed the experience would be from both a staff and a patient
Emotional mapping responses - staff perspective

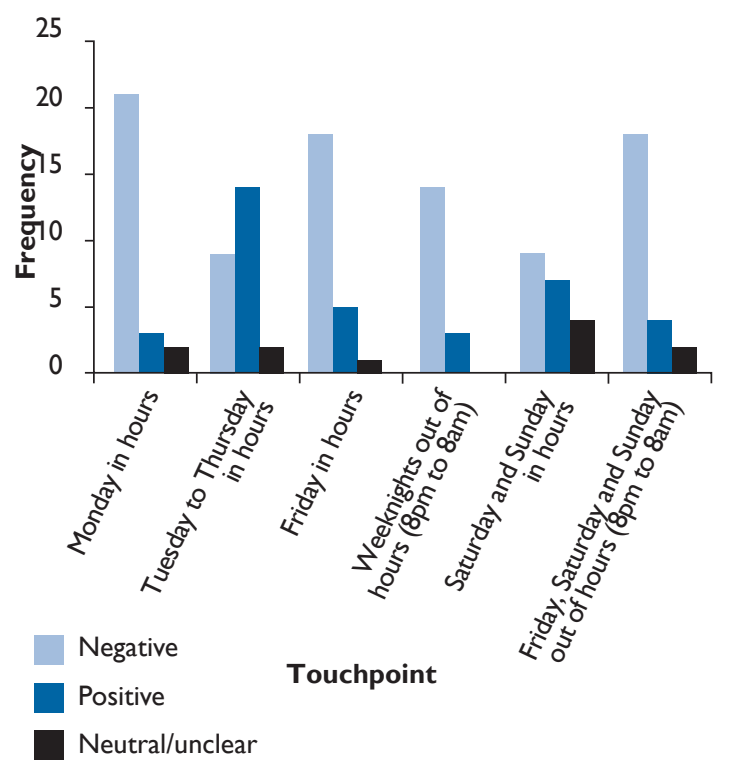

CHART 2 Emotional mapping responses - patient perspective

Emotional mapping responses - patient perspective

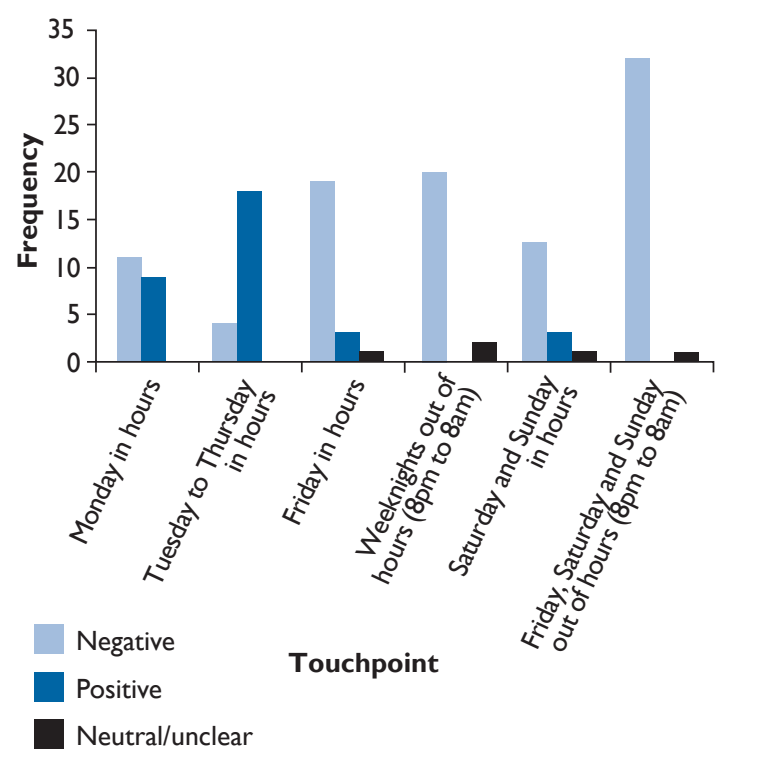

CHART 3 Emotional mapping responses - staff perspective

perspective in an emergency care setting at particular points in time, such as Monday in-hours or weeknights out-of-hours.

This exercise provided an insight into both patient and staff negative anticipation and experience. Interestingly, while the patterns were similar, there were some differences with staff negative perceptions being greatest on Monday and after 8pm at weekends. Both patient and 


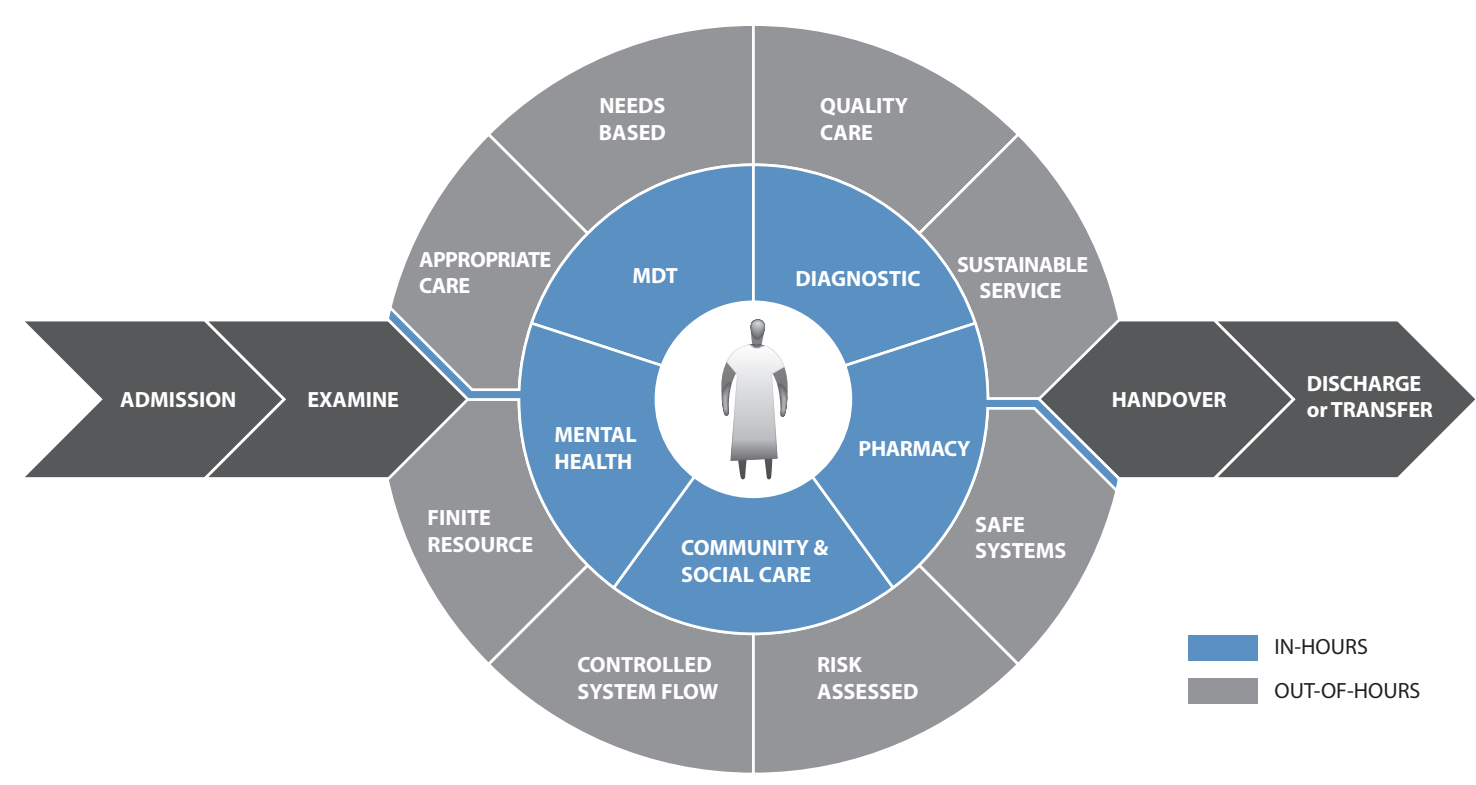

FIGURE I High quality care for patients presenting at acute care services consistently and sustainably, no matter what time they enter the service $24 / 7 / 365$

staff perception was that Friday through Monday and the start of the week is particularly poor, with Tuesday through Thursday in-hours being perceived as the most positive experience. The results suggest that from both a staff and patient perspective there are perceived problems in the patient and staff experience.

Chart I shows an analysis of a Monday to Sunday pattern for the average length of stay and this is also observed in the emotional mapping of staff and patients shown in Charts 2 and 3. The emotional mapping charts depict the projected emotions of staff and patients in acute services, by asking delegates to describe in one word what they perceived staff and patients would feel at different points in time.

There is a clear view that any solutions would involve a broader focus than simply focusing on senior medical staff working weekends. This is evident in the staffing and support services model underpinning the acute medical standards in Appendices A and B which are available via the link at the end. In order to unpack the complexity of the problem and reach a comprehensive understanding of possible targeted solutions, the correct research data and analytical building blocks must be in place.

\section{SUMMARY}

There needs to be a phased approach to providing more consistent acute medical services (Figure I). All services do not need to be $24 / 7$, but an appropriate sustainable solution will require a clear understanding of the contri- buting factors to worse outcomes associated with admission in the out of hours periods. Appropriately targeted but complex interventions will be required to improve outcomes. As such, interventions will have to be prioritised and sequenced with the impact of each monitored while carefully taking into account the following existing factors which are thought to contribute: higher risk of death cases Monday to Friday; staffing levels that do not meet demand or risk profile; and reduced access to diagnostics and specialty out-of-hours.

\section{RECOMMENDATIONS}

- The evidence supports the position that solutions to consistent acute medical care will be multiprofessional and based on the building blocks of robust research, data and analysis

- Acute medical care must continue to address the needs of a patient population that requires complex clinical intervention. Key services include medical, nursing, allied health, pharmacy and diagnostics with appropriate access to specialty, mental health and community and social care

\section{ONLINE CONTENT}

Please follow the link below to view the key points and appendices relating to this paper

https://www.rcpe.ac.uk/policy-standards/ consistent-healthcare-services 


\section{REFERENCES}

I Reed JE, McNicholas C, Woodcock T et al. Designing quality improvement initiatives: the action effect method, a structured approach to identifying and articulating programme theory. BMJ Qual Saf 20I4; 23: I040-8. http://dx.doi.org/I0.I I36/bmjqs-20 I4003103

2 Handel AE, Patel SV, Skingsley A et al. Weekend admissions as an independent predictor of mortality: an analysis of Scottish hospital admissions. BMJ Open 20I2; 2. http://dx.doi.org//0.II36/ bmjopen-2012-001789

3 Freemantle N, Ray D, McNulty D et al. Increased mortality associated with weekend admission: a case for expanded seven day services? BMJ 20I5; 35I. http://dx.doi.org/I0.I I36/bmj.h4596

4 Mikulich O, Callaly E, Bennett $\mathrm{K}$ et al. The increased mortality associated with a weekend emergency admission is due to increased illness severity and altered case-mix. Acute Med 201 I; 10: $181-6$.

5 Aylin P. Making sense of the evidence for the weekend effect. BM] 20I5; 35I. http://dx.doi.org 10.1 I36/bmj.h4652
6 Aiken LH, Sloane DM, Bruyneel L et al. Nurse staffing and education and hospital mortality in nine European countries: a retrospective observational study. Lancet 2014; 383: 1824-30. http://dx.doi.org//0.1016/S0I40-6736(I3)6263I-8

7 Bray B, Ayis S, Campbell J et al. Associations between stroke mortality and weekend working by stroke specialist physicians and registered nurses: prospective multicentre cohort study. PLoS Med 20I4; II: el00I705. http://dx.doi.org/I0.I37I/journal. pmed. 1001705

8 NICE releases safe staffing evidence reviews. I8 January 2016. https:// www.nice.org.uk/news/feature/nice-releases-safe-staffingevidence-reviews

9 Drennan J, Recio-Saucedo A, Pope C et al. Safe Staffing for Nursing in Accident and Emergency Departments Evidence Review. 26 November 2014. https://www.nice.org.uk/guidance/gidsgwave0762/documents/safe-staffing-guidelines-accident-andemergency-departments-evidence-review2 